\title{
Nearby Nature Buffers the Pain Catastrophizing-Pain Intensity Relation Among Urban Residents With Chronic Pain
}

\author{
Nancy M. Wells ${ }^{1 *}$, Kimberly A. Rollings ${ }^{2}$, Anthony D. Ong ${ }^{3}$ and M. Carrington Reid ${ }^{4}$ \\ ${ }^{1}$ Department of Design and Environmental Analysis, College of Human Ecology, Cornell University, Ithaca, NY, United States, \\ ${ }^{2}$ School of Architecture, Department of Psychology, University of Notre Dame, Notre Dame, IN, United States, ${ }^{3}$ Department \\ of Human Development, College of Human Ecology, Cornell University, Ithaca, NY, United States, ${ }^{4}$ Division of Geriatric and \\ Palliative Medicine, Joan and Sanford I. Weill Department of Medicine, Weill Cornell Medical College, New York, NY, \\ United States
}

OPEN ACCESS

Edited by:

Lindsay Joyce McCunn,

Vancouver Island University, Canada

Reviewed by:

Abby Tabor,

University of Bath, United Kingdom

Calia A. Morais,

University of Florida, United States

*Correspondence:

Nancy M. Wells

nmw2@cornell.edu

Specialty section:

This article was submitted to Sustainable Design and Construction, a section of the journal Frontiers in Built Environment

Received: 26 July 2019 Accepted: 15 November 2019 Published: 03 December 2019

Citation:

Wells NM, Rollings $K A$, Ong $A D$ and Reid MC (2019) Nearby Nature Buffers the Pain Catastrophizing-Pain Intensity Relation Among Urban Residents With Chronic Pain.

Front. Built Environ. 5:142. doi: 10.3389/fbuil.2019.00142
Pain catastrophizing is among the strongest predictors of pain intensity. This study examined the role of the nearby natural environment in the experience of pain among community-dwelling adults with chronic pain $(n=81)$ living in New York City and explored the notion that attention may underlie nature's effect. Nearby nature was objectively measured using satellite data. Daily diary data across 14 days was employed to operationalize pain catastrophizing (and subscales: rumination, helplessness, and magnification) and pain intensity. Results indicated that nearby nature buffered the relation between catastrophizing and pain intensity. Moreover, nearby nature moderated the association between pain-related rumination (the most attentionbased subscale of pain catastrophizing) and pain intensity, but did not moderate the helplessness-pain intensity or the magnification-pain intensity associations. These results suggest that the mechanism underlying nearby nature's moderating influence involves attention. Practitioners in search of strategies to reduce pain intensity experienced by community-dwelling chronic pain sufferers might look to a community resource: nearby nature.

Keywords: natural environment, urban, chronic pain, attention, pain catastrophizing, rumination

\section{INTRODUCTION}

Globally, the World Health Organization estimates that chronic pain afflicts one in five adults ${ }^{1}$. An estimated 50-80 million people in the United States experience chronic pain (Gatchel, 2004; Johannes et al., 2010), i.e., pain that lasts beyond the expected time of healing, which is typically 3 months. Estimates of chronic pain prevalence among older adults vary (Holtzman and DeLongis, 2007), but in general, middle-aged and older adults are at increased risk for chronic pain (ReyesGibby et al., 2007; Johannes et al., 2010). Pain catastrophizing_-an exaggerated negative response to actual or anticipated pain characterized by worry, fear, and difficulty directing attention away from pain-is one of the most reliable predictors of the chronic pain experience (Turk and Rudy, 1992; Sullivan et al., 2001). Pain catastrophizing has been linked empirically to the experience of pain across diverse groups of patients, both young and old (for a review, see Sullivan et al., 2001). Pain

\footnotetext{
${ }^{1}$ International Association for the Study of Pain [IASP]. Unrelieved pain is a major global healthcare problem. Retrieved from https://www.iasp-pain.org/files/Content/ContentFolders/GlobalYearAgainstPain2/20042005RighttoPainRelief/ factsheet.pdf.
} 
catastrophizing is associated with lower pain thresholds, greater pain intensity in both clinical and experimental settings, as well as increased pain behavior, greater use of healthcare services, longer hospital stays, and increased use of analgesic medicine (Sullivan et al., 2001; Kjøgx et al., 2016). Given the robust linkage of pain catastrophizing with pain intensity, strategies to reduce the experience of pain within community settings (Reid et al., 2008; Tobias et al., 2014) might aim to uncouple the link between pain catastrophizing and pain intensity. This study examines the potential of an environmental factor-nearby nature-to diminish the strength of association between pain catastrophizing and pain intensity.

Why might nature affect pain intensity? This hypothesized association is supported by both theory and empirical evidence from several domains. We begin by reviewing evidence linking the natural environment to health, in general, and to pain, specifically. Next, we consider research documenting the central role of attention in the experience of pain. We then briefly describe the principles of Attention Restoration Theory (ART), a theoretical perspective purporting that exposure to the natural environment restores ability to direct and control attention, and we present related empirical evidence that nature enhances cognitive control. Finally, we test our hypotheses using data drawn from a study of community-dwelling middle-aged and older adults residing in an urban environment.

Many studies have documented an association between the prevalence of nature in residential environments and general health indicators (Frumkin, 2001; Takano et al., 2002; Groenewegen et al., 2006; Maas et al., 2006, 2009; Mitchell and Popham, 2007; Wells and Rollings, 2012; Hartig et al., 2014; Capaldi et al., 2015; James et al., 2015; Frumkin et al., 2017; Wells and Phalen, 2018). Prisoners with natural views from their windows made fewer visits to the infirmary than those with built views (Moore, 1981; West, 1986); other studies have examined the therapeutic role of nature in healthcare settings (e.g., Verderber and Reuman, 1987; Ulrich, 1999; for reviews see: Irvine and Warber, 2002); however, few studies have examined the relation between exposure to nature and pain intensity. One early study employed archival medical record data of patients who had undergone gallbladder surgery to examine the relation of hospital room window view (trees vs. brick wall) and recovery from surgery (Ulrich, 1984). Compared to patients with a brick wall view, those with views of nature not only had shorter postoperative stays in the hospital and fewer negative notes in their files from the nurses but also took fewer doses of analgesic medications in the post-operative period. These findings raise the questions of why and how might nature be associated with reduced pain? Attention may provide an answer.

\section{Attention and Pain}

\footnotetext{
"Usual uses of the term attention often underemphasize the controlling role of the environment in favor of the attentiondirecting role of the individual. Reversing this relationship appears to have heuristic value for the study of chronic pain. ..." (McCracken, 1997, p. 280).
}

"The future of the field of pain... lies in understanding the role of the brain" (Melzack, 1999, p. 881).

Studies indicate that attention plays a key role in pain (McCracken, 1997; Eccleston and Crombez, 1999). This work is based on the notion that attention is a limited resource and that perceived pain is a function of the amount of attention devoted to pain (McCracken, 1997). Evidence suggests that pain demands cognitive resources and hinders the individual from directing attention elsewhere. Eccleston (1995), for example, demonstrated that patients suffering from chronic pain were more impaired in their performance of an attentionally-demanding task, compared to patients with low levels of pain and normal controls.

Rather than studying how pain draws attentional resources away from other endeavors, some studies have explored how intentional distraction from pain (by attending to other stimuli) might increase tolerance of pain or reduce pain intensity. Researchers have examined the effect of distraction on pain by either providing stimuli to patients undergoing a potentially painful medical procedure or by exposing pain-free participants to acute pain in a laboratory setting with or without distraction. For example, Arntz et al. (1994) found that compared to subjects in the distraction condition (i.e., who watched an episode of the television program "L.A. Law"), those in the attention condition (i.e., who were instructed to concentrate as much as possible on the pain stimulus) rated the pain of electrical stimulation much higher. Similarly, Arntz et al. (1991) documented that attention to pain, rather than anxiety, affected pain impact. Interestingly, numerous "distraction therapy" studies have used nature stimuli to examine the role of visual and/or auditory stimulation as a strategy to reduce the discomfort associated with flexible sigmoidoscopy (Lembo et al., 1998), colonoscopy (Lee et al., 2004), burn dressing change (Miller et al., 1992), flexible bronchoscopy (Diette et al., 2003), and induced pain by submerging participants' hands in cold water (Park et al., 2004; for review, see: Kline, 2009). Remarkably, although all these studies employ nature stimuli [e.g., "ocean scenes and sounds" (Lembo et al., 1998); "mainly scenic views" (Lee et al., 2004), a video containing “... ocean, desert, forest, flowers, waterfalls, and wildlife" (Miller et al., 1992); foliage and flowering plants (Park et al., 2004)] to explore the notion of "distraction therapy," few studies explicitly consider the potential importance of the specific content of their intervention stimuli: nature (i.e., Diette et al., 2003; Kline, 2009).

Additional evidence for the role of attention in pain is provided by investigations of pain catastrophizing, and, in particular the rumination component of catastrophizing. Rumination is a preoccupation with pain, when a person attends to or "keeps thinking" about the pain and "can't seem to keep it out of [their] mind." Findings indicate that of the three subscales of the pain catastrophizing scale (i.e., magnification, rumination, helplessness), rumination is the strongest predictor of pain intensity (Sullivan and Neish, 1998; Sullivan et al., 2001).

\section{Nature and Attention}

Attention Restoration Theory (ART) (Kaplan, 1995, 2001) provides a conceptual framework for understanding how nature 
affects attention, and in turn, how this association might be relevant to the pain experience. Building on the work of James (1892), ART proposes that attention consists of two components: voluntary or "directed" attention, which requires effort as neural inhibitory mechanisms block out potential distraction to allow focus and concentration; and involuntary attention, which is captured effortlessly. When involuntary attention is gently engaged, inhibitory mechanisms underlying directed attention are able to rest and recover, restoring attentional control and focus. Environments rich in inherently fascinating stimuli easily engage involuntary attention or "fascination," allowing directed attention to recover. Natural settings and stimuli tend to be particularly effective at engaging involuntary attention. Thus, exposure to nature enhances attentional capacity. This theory has been substantiated by a wide variety of empirical research, which we briefly summarize below.

In recent decades, evidence that nature reduces directed attention fatigue and enhances attentional capacity has been found among a variety of subjects. College students with dormitory views of trees and grass performed better on cognitive tasks than did students with views predominantly of buildings, cars, and streets (Tennessen and Cimprich, 1995). Backpackers who took a wilderness hiking vacation showed more improvement (pre- to post-vacation) on a proof-reading task than did others who were assigned to a non-wilderness vacation or no vacation (Hartig et al., 1991, Study 1). Cognitive functioning improvements among children whose families relocated were explained by increases in neighborhood greenness (Wells, 2000). Children diagnosed with attention deficit disorder (ADD) have been shown to have fewer symptoms in green settings (Faber Taylor et al., 2001; Faber Taylor and Kuo, 2009). Intervention studies indicate that nature bolsters the cognitive functioning of women recently diagnosed with breast cancer (Cimprich, 1992; Cimprich and Ronis, 2003). Students randomly assigned to take a nature walk after completing cognitivelyfatiguing tasks performed better on a subsequent cognitive task than did others assigned to take an urban walk, or to quietly relax indoors (Hartig et al., 1991, study 2). Similarly, students exposed to images of nature recovered from induced directed attention fatigue, whereas those who saw urban images did not (Berto, 2005). Adding to this evidence, Berman et al. (2008) document the specific components of attention that are affected by nature exposure. The researchers used a within-subjects design, assigning subjects to either a nature walk or an urban walk of the same distance. Comparing performance on the digit span backwards task (DSB) after the walk to before the walk, DSB improved significantly when participants walked in nature, but not when they walked on urban streets. In Study 2, participants were shown images of either nature scenery or urban settings and along with DSB, the Attention Network Test (ANT) (Fan et al., 2002, 2005) was used to examine three attentional processes: alerting, orienting, and executive attention. As hypothesized, nature affected only the ANT process that most involves directed attention: executive attention; but had no effect on alerting or orienting. DSB findings replicated those of Study 1. Moreover, research evidence also links nature experience to reductions in rumination among healthy adults (Bratman et al., 2015).
While most of these studies operationalize attentional capacity with a task requiring focus and concentration, a few studies measure attention not only with concentration tasks, but also with tasks requiring impulse control or inhibition. Girls living in large, urban public housing buildings with green, treed apartment views performed better than girls with barren views in activities related to concentration, impulse inhibition, and delay of gratification (Faber Taylor et al., 2002). These findings suggest that nearby nature is a resource that bolsters cognitive capacity, enabling young girls living in the context of poverty and crime to inhibit prepotent or impulsive responses. A second study in Chicago public housing suggests that nearby nature may have similar benefits for low income women. Findings indicate that women with green, natural views performed better on measures of cognitive functioning (DSB) and were more able to manage major life issues. Moreover, cognitive functioning was found to mediate the relation between nearby nature and ability to manage major life issues (Kuo, 2001).

Although ART has not previously been employed to examine the influence of nature on pain catastrophizing and pain intensity, ART provides a compelling theoretical framework for this topic. Given that exposure to nature enhances one's capacity to control or direct attention, and to resist the pull of competing demands (suppress distracting stimuli), it is reasonable to ask whether access to nearby nature enables a person to direct attention away from unproductive ruminations (including the distraction of pain) and thereby reduces pain catastrophizing and pain intensity.

\section{Summary}

Empirical evidence suggests that nature reduces pain intensity (Ulrich, 1984); that pain draws upon attentional resources (McCracken, 1997; Sullivan and Neish, 1998; Eccleston and Crombez, 1999; Sullivan et al., 2001) limiting capacity to perform cognitive tasks (Arntz et al., 1994; Eccleston, 1995); various stimuli, including nature, draw attention away from pain, reducing the pain intensity experience (Arntz et al., 1991; Miller et al., 1992; Lembo et al., 1998; Diette et al., 2003; Lee et al., 2004; Park et al., 2004) (for review, see Kline, 2009). Moreover, it is welldocumented that nature enhances attentional capacity, reduces directed attention fatigue, allows greater focus and cognitive control (Hartig et al., 1991; Cimprich, 1992; Tennessen and Cimprich, 1995; Wells, 2000; Faber Taylor et al., 2001, 2002; Cimprich and Ronis, 2003; Berto, 2005; Berman et al., 2008) and reduces rumination (Bratman et al., 2015).

\section{The Present Study}

Leveraging a previously assembled sample of middle-aged and older adults with chronic pain, this study examines the notion that nearby nature may help to reduce pain intensity by disrupting the linkage between pain catastrophizing and pain intensity. Second, if attention specifically is affected, we expect that the relation between rumination, the most attention-related subscale of pain catastrophizing (as opposed to helplessness or magnification subscales) and daily pain intensity will be diminished among those with more nearby nature. Accordingly, two research questions are examined in this study (Figures 1, 2). 

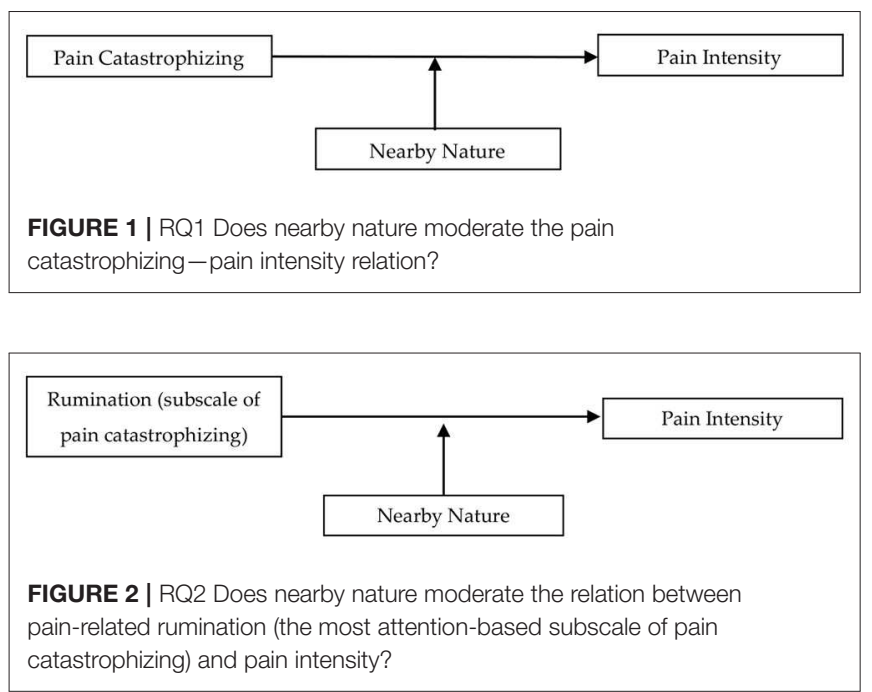

Research Question 1 (RQ1) asks: Does nearby nature moderate the association of pain catastrophizing and daily pain intensity? Research Question 2 (RQ2) examines: Does nearby nature moderate the association between rumination and daily pain intensity, but not the relations between helplessness and pain intensity, and between magnification and pain intensity?

\section{MATERIALS AND METHODS}

\section{Participants}

Ninety-six New York City metropolitan area residents were recruited from patients enrolled in an academically-affiliated primary care practice in the city. Physicians referred patients with chronic pain diagnoses to participate in the study. Chronic pain was defined as "the presence of pain, aching, burning, or throbbing on most days of every month for at least three consecutive months in the past year due to a health condition other than cancer" (Ong et al., 2010, p. 517). Within this sample, chronic pain was most commonly due to lower back pain and osteoarthritis of the hip and/or knee. Physicians' diagnoses of chronic pain were supplemented with inclusionary and exclusionary criteria based on participant reports of pain. Only those patients diagnosed with chronic pain who reported a pain level $>5$ on a rating scale $(0=$ no pain, $10=$ pain as bad as you can imagine) during the week prior to the beginning of the study were included in the sample. Fifteen participants were excluded from this analysis-two because they provided only post office box mailing addresses, which prevented objective measurement of nature surrounding their physical residence, and 13 who did not provide complete baseline information. The analytic sample consisted of 81 participants whose sociodemographic characteristics are displayed in Table $\mathbf{1 .}$

\section{Procedure}

All data collection procedures were approved by institutional review boards at Cornell University and Weill Cornell Medical College and are documented in the parent study (Ong et al., 2010). Upon meeting study criteria, participants signed
TABLE 1 | Participant socio-demographic variables.

\begin{tabular}{|c|c|c|c|}
\hline Variable & Levels & \# & $\%$ \\
\hline \multirow[t]{3}{*}{ Ethnicity } & Caucasian & 77 & 95.1 \\
\hline & Hispanic/Latino & 1 & 1.2 \\
\hline & African American & 3 & 3.7 \\
\hline \multirow[t]{2}{*}{ Gender } & Male & 21 & 25.9 \\
\hline & Female & 60 & 74.1 \\
\hline \multirow[t]{4}{*}{ Age } & $50-59$ & 5 & 6.2 \\
\hline & $60-69$ & 10 & 12.3 \\
\hline & $70-79$ & 40 & 49.4 \\
\hline & $80+$ & 26 & 32.1 \\
\hline \multirow[t]{7}{*}{ Income } & $<\$ 7,500$ & 3 & 3.7 \\
\hline & $\$ 7,500-14,999$ & 12 & 14.8 \\
\hline & $\$ 15,000-24,999$ & 11 & 13.6 \\
\hline & $\$ 25,000-39,999$ & 9 & 11.1 \\
\hline & $\$ 40,000-74,999$ & 15 & 18.5 \\
\hline & $\$ 75,000-99,999$ & 11 & 13.6 \\
\hline & $>\$ 100,000$ & 20 & 24.7 \\
\hline \multirow[t]{5}{*}{ Marital status } & Single & 19 & 23.5 \\
\hline & Separated & 1 & 1.2 \\
\hline & Divorced & 13 & 16.0 \\
\hline & Married & 27 & 33.3 \\
\hline & Widowed & 21 & 25.9 \\
\hline \multirow{5}{*}{$\begin{array}{l}\text { Living companion } \\
\text { (1 unreported) }\end{array}$} & Alone & 41 & 50.6 \\
\hline & Spouse & 24 & 29.6 \\
\hline & Friend & 2 & 2.5 \\
\hline & Children & 7 & 8.6 \\
\hline & Other & 6 & 7.4 \\
\hline \multirow[t]{7}{*}{ Education level } & Middle school & 5 & 6.2 \\
\hline & High school & 11 & 13.6 \\
\hline & Vocational ed & 1 & 1.2 \\
\hline & Some college & 14 & 17.3 \\
\hline & College degree & 21 & 25.9 \\
\hline & Post-college prof degree & 17 & 21.0 \\
\hline & Grad, med, law degree & 12 & 14.8 \\
\hline
\end{tabular}

written consent forms and were given initial baseline sociodemographic and personality measure questionnaires which they returned by mail. Participants then received 14 daily diary questionnaires containing pain measures, instructions for operating an electronic stamper used to seal each day's responses to insure daily diary completion, and 14 postage-paid envelopes. Researchers phoned participants with instructions before they completed the diaries one half-hour before bedtime each day, and phoned weekly to answer any questions and remind participants to complete the diaries. Participants sealed each day's responses in an addressed envelope and stamped the seal with a hand-held electronic time stamper (DYMO Corp., Stamford, CT) provided by the researchers. The stamper imprinted the current date and time and was programmed such that the date and time could not be altered. Participants were compensated $\$ 75$ for participation. Use of the time stamper to monitor diary completion, along with weekly phone calls, and monetary incentives resulted in a high compliance rate that did not differ by gender or age. Of a 
possible 1,330 time-stamped daily diaries, participants completed the diary on 1,237 days (93\%); $85 \%$ of the completed diaries were completed on time (i.e., on the correct night or before noon the following day). The modal completion time was 8:00 p.m., with $96 \%$ of the diary checklists completed between $6: 00$ p.m. and 12:00 a.m. Analyses examining only diaries completed on time revealed no significant difference from analyses using all completed diaries, regardless of when they were completed. Final analyses, therefore, are based upon analyses using all completed diaries.

\section{Constructs and Measures \\ Daily Measures (Level 1)}

The dependent variable, daily pain intensity, was reported daily by participants for 14 days. Participants were asked to rate the pain they experienced during the current day by choosing a number between 0 and 10 that "best describes your average level of pain today: A zero (0) indicated 'no pain' while a ten (10) meant 'pain as intense as you can imagine"' (Jensen et al., 1999, p. 159). Reliability was estimated via test-retest correlations across weeks, which resulted in a week-to-week correlation of 0.72 .

The independent variables measured daily for 14 days and used in this analysis were: pain catastrophizing, rumination, helplessness, and magnification. Pain catastrophizing, an exaggerated, negative response to actual or anticipated pain, is characterized by worry, fear, and difficulty directing attention away from pain, and is one of the most reliable predictors of the chronic pain experience (Turk and Rudy, 1992; Sullivan et al., 1995; Ong et al., 2010). Participants' pain catastrophizing was measured by summing responses to seven statements representing three related but distinct subscales of pain catastrophizing: rumination, helplessness, and magnification (Sullivan et al., 2001). Participants selected a number from one (not at all) to five (all the time), indicating the extent to which they experienced the feelings described in each of the seven items. To minimize participant burden, we employed a 7-item scale of pain catastrophizing, rather than the original 13 -item scale. Daily pain catastrophizing was measured using items with the highest total correlations from past research (Sullivan et al., 1995). Internal consistency reliability of this 7-item measure is 0.91 as reported in the parent study (Ong et al., 2010). Rumination is the most attention-related subscale, and is most relevant when examining the effects of nearby nature on pain. The rumination subscale consisted of three of the seven pain catastrophizing statements: "I anxiously wanted the pain to go away," "I can't seem to keep the pain out of my mind," and "I kept thinking about how badly I wanted the pain to stop." Rumination was measured as the sum of responses from these three items.

To account for variations in participant exposure to nearby nature and to isolate its effects, daily time in nature was included as a control variable. The variable was operationalized as the total time $(0,1-15,16-30 \mathrm{~min}, 30 \mathrm{~min}-1 \mathrm{~h}, 1-2 \mathrm{~h}$, more than $2 \mathrm{~h}$ ) participants reported that they viewed, sat, walked, or jogged outdoors in a green grassy area, meadow or field, forest or treed woods, flower or vegetable garden, or other outdoor environment each day. Reliability was estimated via test-retest correlations across weeks, which resulted in a week-to-week correlation of 0.47 .

\section{Person-Level Measures (Level 2)}

Residential nearby nature was an objectively measured, personlevel independent variable that provided an indication of how much nature was proximate to each participant's home address. Using Google Maps, a satellite map view of each participant's address, generated using the same computer monitor to maintain a constant image resolution and extent, was set to the $50^{\prime}(20 \mathrm{~m})$ viewing scale. Once the image was centered on the address, a one-inch grid ( 10 one-inch squares wide $\times 5$ squares tall, or 500 by 250 feet) was digitally superimposed on the saved map. The amount of nearby nature was determined by first estimating the percentage of nature (water, trees, green space) within each grid square $(0,10,25,50,75,90$, or $100 \%)$, and then calculating the average percentage of nature within the map view. The overall inter-rater reliability (Ebel) of this measure, based on three researchers' estimations of nearby nature for 30 addresses, was 0.997 . This measure was used because land cover data often does not distinguish between varying amounts of natural areas in dense urban areas. Additionally, tall building shadows prevent some trees, grass, and other vegetation from being captured in satellite images, yielding lower estimates of natural areas. Other available GIS data containing park, open space, and tree locations also yield low estimates of natural areas because they exclude privately owned land that could also contain additional natural areas, especially surrounding residential locations. Typical areas of low, medium, and high nearby nature in the New York City area are displayed in Figure 3.

Because individuals may differ in their ability to capitalize on positive emotions during stressful events such as the experience of chronic pain (Ong et al., 2010), psychological resilience was controlled for in this analysis. Psychological resilience was measured by the 14-item Ego-Resiliency Scale (Block and Kremen, 1996) included with baseline questionnaires, and indicated "the capacity of the individual to effectively modulate and monitor an ever-changing complex of desires and reality constraints"(Block and Kremen, 1996, p. 359). Responses were made on a 4-point scale, ranging from 1 (does not apply at all) to 4 (applies very strongly). Sample items include "I am regarded as a very energetic person" and "I get over my anger with someone reasonably quickly." Responses were then averaged, yielding a psychological resilience score. For this sample, internal consistency reliability (Cronbach alpha) was 0.78 and has been reported previously as 0.76 (Block and Kremen, 1996).

Neuroticism was also controlled for in this analysis. The Mini-IPIP (20-items from the 50-item International Personality Item Pool or IPIP) contained a four-item neuroticism scale that was used to measure person-level neuroticism (Donnellan et al., 2006). Each item, such as, "I have frequent mood swings," was responded to on a 5-point Likert scale, ranging from 1 (strongly disagree) to 5 (strongly agree). Responses to all four items were then averaged. For this sample, the reliability (Cronbach alpha) was 0.76 ; Donnellan et al. (2006) report alphas ranging from 0.68 to 0.70 . 


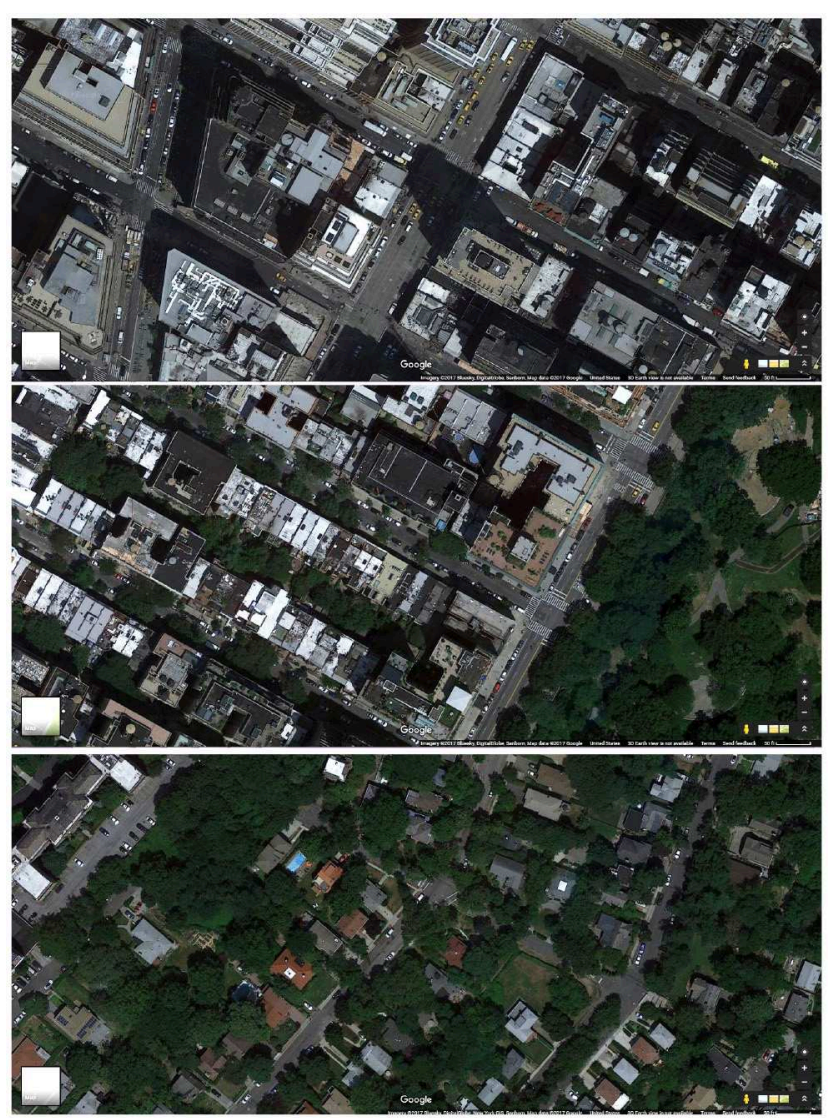

FIGURE 3 | Prototypical low (top), medium (middle) and high (bottom) nearby nature in the New York City area (Imagery: (2017 Bluesky, DigitalGlobe, New York GIS, Sanborn, Map data: (2017 Google, used under license).

Age, gender (males $=0$, females $=1$ ), and income were also measured at baseline and controlled for in this analysis. Age was a continuous variable, while income was categorical: < < $\$ 7,500 ; \$ 7,500-14,999 ; \$ 15,000-24,999 ; \$ 25,000-$ 39,$000 ; \$ 40,000-74,999 ; \$ 75,000-99,999$; and $>\$ 100,000 . \$ \$$

\section{Overview of Data Structure and Analyses}

This study's two-part analysis examined objectively-measured nearby nature as a moderator of the associations between pain catastrophizing and pain intensity (RQ1), and between rumination and pain intensity (RQ2). The multilevel data structure consisted of repeated daily measures (up to 14 consecutive days) nested within 81 persons. Multilevel models are appropriate for repeated measures data because multiple observations of the same person lack independence. Coefficients estimated for each Level 1 unit of analysis are analyzed at Level 2 as dependent variables in a two-level model (Nezlek, 2001). In this study, the daily measures can be analyzed withinsubjects at Level 1 and between-subjects at Level 2. The Level 1 daily measures [pain catastrophizing (rumination, helplessness, magnification), time in nature] are time-varying and differ across the 14 days within each participant. The Level 2 person-level variables (nearby nature, psychological resilience, neuroticism, age, gender, and income) are constant or time-invariant and vary only between participants throughout the study.
The current study examined two multilevel models. The first (RQ1) examined whether nearby nature moderated the pain catastrophizing and pain intensity relation. The second model (RQ2) explored whether nearby nature moderated the relation between the rumination subscale of pain catastrophizing, specifically, and pain intensity. In each model, a Level 1 by Level 2 interaction term between pain catastrophizing (RQ1), rumination (RQ2), and nearby nature was added to examine the hypothesized moderation. Hierarchical Linear Modeling software (Version 6.0; Raudenbush et al. (2011) was used to model the two, two-level equations. The full Level-1 and Level-2 models, including all independent variables and control variables, are provided in Supplementary Material.

As control variables, neuroticism, resilience, age (continuous), gender, and income (seven levels as illustrated in Table 1) were modeled as fixed effects and grand-mean centered (indicated by italics within equations). This centering calculates an overall average value for each of these variables across the 14 study days and all participants. Catastrophizing, rumination, helplessness, magnification, and time in nature were all modeled as random effects.

\section{RESULTS}

\section{RQ1: Pain Intensity and Pain Catastrophizing}

Table 2 displays results of the first multi-level model (RQ1), including final estimation of fixed effects and variance components (random effects). Intercept or fixed effect coefficients $\left(\beta_{00}-\beta_{06}, \beta_{10}, \beta_{20}\right)$ that are significant indicate main effects on pain intensity. Pain catastrophizing was a significant predictor of pain intensity $\left(\beta_{10}=0.27, p<0.001\right)$. The main effect of nearby nature on pain intensity was non-significant $\left(\beta_{01}=-0.02, p=0.051\right)$. Other coefficients are associated with catastrophizing $\left(\beta_{11}-\beta_{16}\right)$ and time in nature $\left(\beta_{21}-\beta_{26}\right)$ interaction terms. Nearby nature significantly moderated the pain catastrophizing-pain intensity relation $\left(\beta_{11}=-0.006\right.$, $p<0.001)$. For each unit increase in the percentage of nearby nature (e.g., from 50 to $51 \%$ ), the association between pain catastrophizing and pain intensity weakened by 0.006 when controlling for psychological resilience, neuroticism, gender, age, and income. To aid in the interpretation, parameter values were generated using values one standard deviation $(S D)$ above and below the mean to represent high and low scores for pain catastrophizing and nearby nature (see Figure 4). Estimates of simple slopes from the two-way multilevel interaction (Bauer and Curran, 2005) confirmed that nearby nature was associated with significantly lower levels of pain on days characterized by high $(b=-0.051, S E=0.01, p<0.001)$ but not low $(b$ $=0.020, S E=0.01, n s)$ pain catastrophizing. Time in nature neither predicted daily pain intensity $\left(\beta_{20}=-0.05, p>0.05\right)$, nor interacted with nearby nature $\left(\beta_{21}=-0.001, p>0.05\right)$ to affect pain intensity.

Unlike fixed effects that examine intercepts, random effects or variance components examine slopes. The pain catastrophizing slope, or random effect, was also significant, indicating that there was significant variance in pain catastrophizing at Level 2 or between-subjects to be explained $\left(\chi^{2}=140.56, p<0.001\right)$. 
TABLE 2 | RQ1: Multilevel regression predicting daily pain intensity including catastrophizing.

Final estimation of fixed effects (with robust standard errors)

\begin{tabular}{|c|c|c|c|c|c|}
\hline Fixed effect & Estimate & SE & T-ratio & d.f. & $p$-value ${ }^{a}$ \\
\hline \multicolumn{6}{|l|}{ For intercept, $\pi_{0 i}$} \\
\hline$\beta_{00}$ intercept & 3.4157 & 0.3220 & 10.607 & 74 & 0.000 \\
\hline$\beta_{01}$ nearby nature & -0.0159 & 0.0080 & -1.984 & 74 & 0.051 \\
\hline$\beta_{02}$ resilience & -0.5078 & 0.5425 & -0.936 & 74 & 0.353 \\
\hline$\beta_{03}$ neuroticism & -0.0437 & 0.4061 & -0.108 & 74 & 0.915 \\
\hline$\beta_{06}$ income & -0.1668 & 0.1191 & -1.401 & 74 & 0.166 \\
\hline \multicolumn{6}{|c|}{ For catastrophizing, $\pi_{1}$} \\
\hline$\beta_{10}$ intercept & 0.2690 & 0.0331 & 8.1250 & 74 & $<0.001$ \\
\hline$\beta_{11}$ nearby nature & -0.0056 & 0.0010 & -5.360 & 74 & $<0.001$ \\
\hline$\beta_{12}$ resilience & 0.0564 & 0.0512 & 1.103 & 74 & 0.274 \\
\hline$\beta_{16}$ Income & 0.0275 & 0.0116 & 2.381 & 74 & 0.020 \\
\hline \multicolumn{6}{|l|}{ For time in nature, $\pi_{2}$} \\
\hline$\beta_{20}$ intercept & -0.0484 & 0.0253 & -1.915 & 74 & 0.059 \\
\hline$\beta_{21}$ nearby nature & -0.0011 & 0.0007 & -1.607 & 74 & 0.112 \\
\hline$\beta_{22}$ resilience & -0.0288 & 0.0310 & -0.929 & 74 & 0.356 \\
\hline$\beta_{23}$ neuroticism & 0.0894 & 0.0283 & 3.161 & 74 & 0.003 \\
\hline$\beta_{24}$ gender & -0.0067 & 0.0335 & -0.200 & 74 & 0.843 \\
\hline$\beta_{25}$ age & -0.0026 & 0.0020 & -1.293 & 74 & 0.200 \\
\hline$\beta_{26}$ income & 0.0157 & 0.0098 & 1.607 & 74 & 0.112 \\
\hline
\end{tabular}

Final estimation of variance components

\begin{tabular}{|c|c|c|c|c|c|}
\hline Random effect & SD & Variance component & d.f. & Chi-square & $p$-value \\
\hline Intercept $r_{0}$ & 1.6330 & 2.6668 & 73 & 319.2644 & $<0.001$ \\
\hline Catastrophizing slope $r_{1}$ & 0.1233 & 0.0152 & 73 & 140.5581 & $<0.001$ \\
\hline Time in nature slope $r_{2}$ & 0.0853 & 0.0073 & 73 & 104.8853 & 0.009 \\
\hline Level-1 e & 1.4686 & 2.1567 & & & \\
\hline
\end{tabular}

a Bolded p-values indicate significance at the 0.05 alpha level.

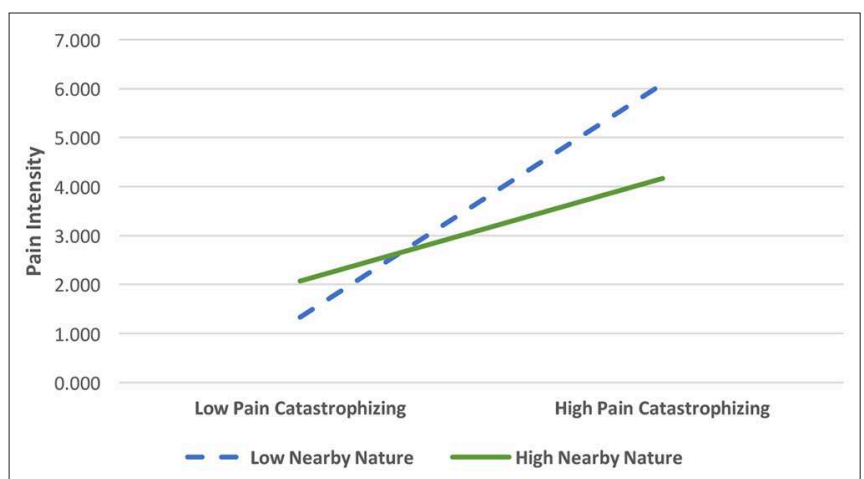

FIGURE 4 | Relationship between pain catastrophizing and pain intensity as a function of nearby nature. High and low values were defined as one standard deviation from the mean.
The pain intensity model that included pain catastrophizing accounted for $23.9 \%$ of the within- and $28.9 \%$ of the betweenperson variance in pain intensity, calculated by comparing variances between fully conditional and unconditional models. These percentages are proxies for effect sizes. Nearby nature accounted for 0.26 and $0.21 \%$ of the within- and between-person variances, respectively. The control variables (time in nature, psychological resilience, neuroticism, gender, age, and income) accounted for an additional 6.9 and $7.3 \%$ of the within- and between-person variances.

\section{RQ2: Pain Intensity and Rumination, Helplessness and Magnification}

Table 3 contains results of the second multi-level model (RQ2). Similar to the first model, intercept or fixed effect coefficients $\left(\beta_{00}-\beta_{06}, \beta_{10}, \beta_{20}, \beta_{30}, \beta_{40},\right)$ that are significant indicate main 
TABLE 3 | RQ2: Multilevel regression predicting daily pain intensity including rumination, magnification, helplessness.

Final estimation of fixed effects (with robust standard errors)

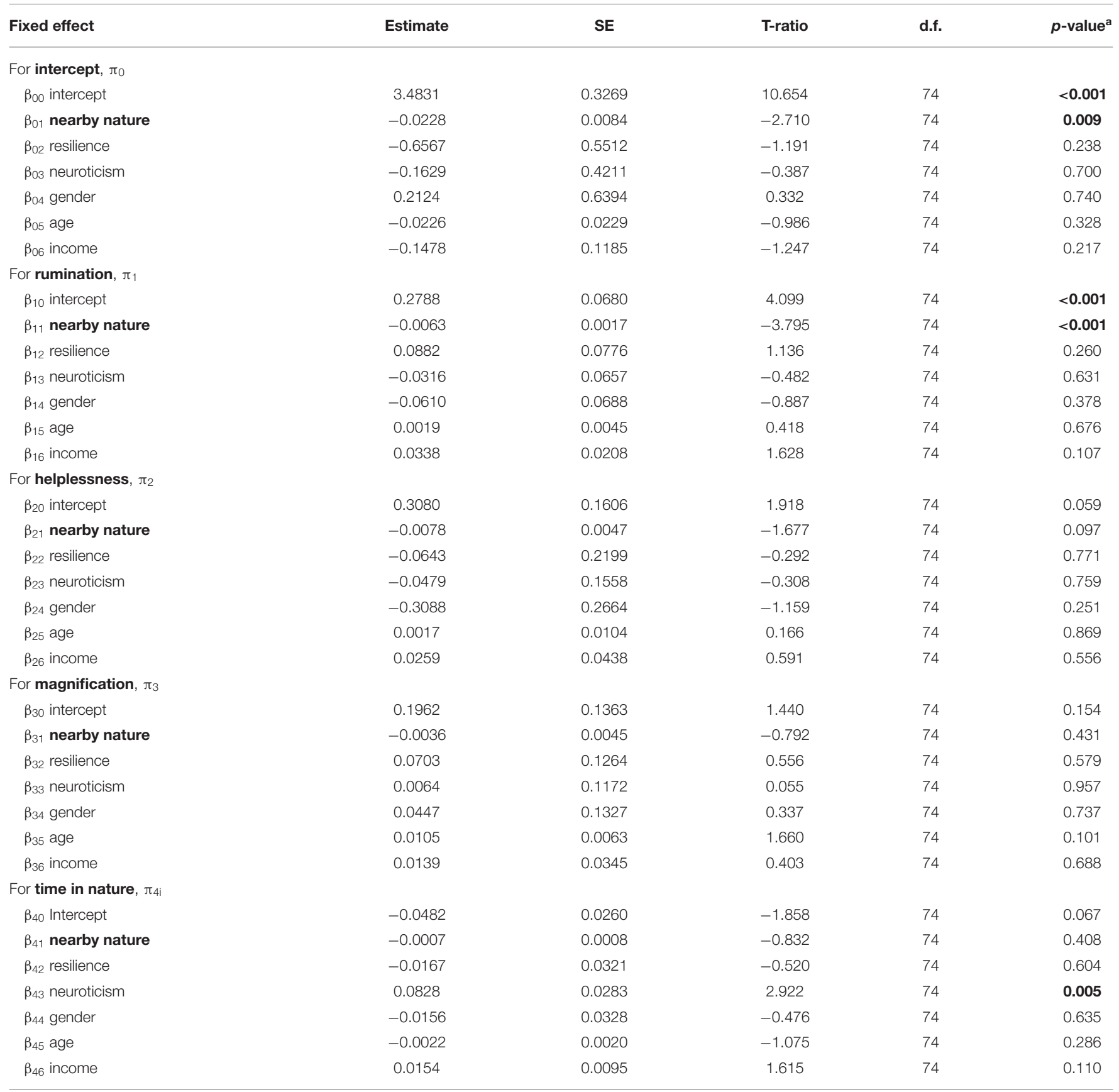

Final estimation of variance components

\begin{tabular}{|c|c|c|c|c|c|}
\hline Random effect & SD & Variance component & d.f. & Chi-square & $p$-value \\
\hline Intercept $r_{0}$ & 1.5729 & 2.4741 & 55 & 175.4792 & 0.000 \\
\hline Rumination slope $r_{1}$ & 0.1608 & 0.0258 & 55 & 57.9279 & 0.368 \\
\hline Helplessness slope $r_{2}$ & 0.6105 & 0.3727 & 55 & 143.8126 & 0.000 \\
\hline Magnification slope $r_{3}$ & 0.2569 & 0.0660 & 55 & 42.9983 & $>0.500$ \\
\hline Time in nature slope $r_{4}$ & 0.0871 & 0.0076 & 55 & 80.5423 & 0.014 \\
\hline
\end{tabular}

${ }^{a}$ Bolded p-values indicate significance at the 0.05 alpha level. 


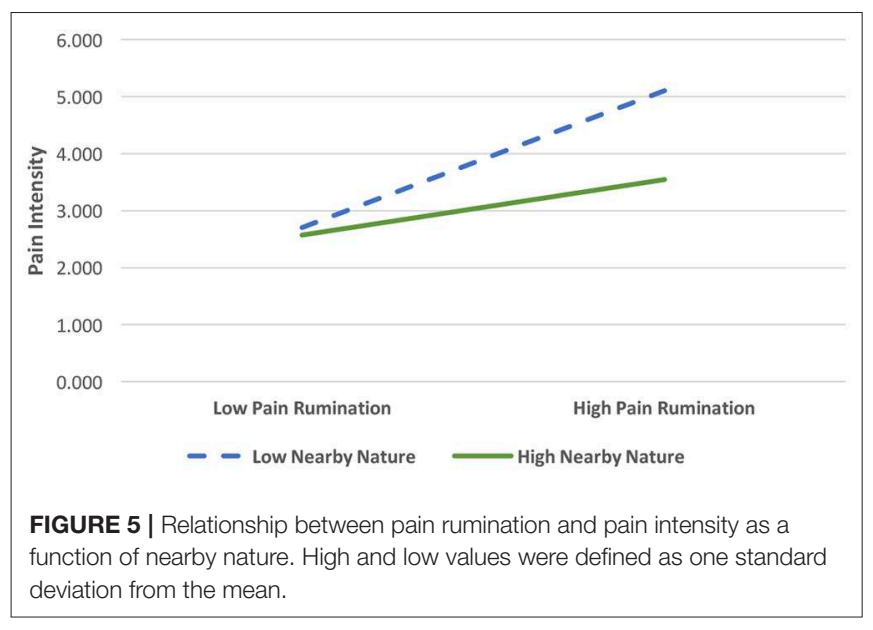

effects on pain intensity. Other fixed effects coefficients are associated with interactions between Level 1 and Level 2 predictors. Significant main effects of rumination $\left(\beta_{10}=0.28\right.$, $p<0.001)$ and nearby nature $\left(\beta_{01}=-0.02, p=0.009\right)$ on pain intensity were found. Consistent with the RQ2 hypothesis, nearby nature moderated the rumination-pain intensity relation, but not the magnification-pain intensity or the helplessness-pain intensity relations. For every unit increase in nearby nature, the association between rumination and pain intensity weakened by $0.006\left(\beta_{11}=-0.006, p<0.001\right)$. Significant main effects of rumination and the interaction between rumination and nearby nature were independent of neuroticism, psychological resilience, gender, age, and income. As depicted in Figure 5, the simple slopes confirmed that on high rumination days $(b=-0.042, S E$ $=0.01, p<0.001$ ), variation in nearby nature was significantly associated with lower pain, but there was no such association on low rumination days $(b=-0.004, S E=0.01, n s)$. Time in nature neither predicted daily pain intensity $\left(\beta_{40}=-0.05, p>\right.$ $0.05)$, nor interacted with nearby nature $\left(\beta_{41}=-0.001, p>0.05\right)$. The rumination variance component was also not significant, indicating that there was no significant Level 2 variance to be explained.

The fully conditional pain intensity model that included rumination accounted for $31.0 \%$ of the within- and $34.0 \%$ of the between-person variances in pain intensity when compared to the fully unconditional model. Nearby nature accounted for 0.1 and $0.8 \%$ of the within- and between-person variances, respectively. The control variables (time in nature, psychological resilience, neuroticism, gender, age, and income) accounted for an additional 7.9 and $7.8 \%$ of the within- and between-person variances. Final estimations of fixed effects with robust standard errors and variance components are displayed in Table 3.

\section{DISCUSSION}

This study represents a preliminary foray into nature's relationship to pain intensity among chronic pain sufferers living in an urban environment. Consistent with the hypotheses, we find that nearby nature moderates the effect of pain catastrophizing on pain intensity; and more specifically, that nearby nature moderates the relation between pain-related rumination and pain intensity. By buffering the impact of pain catastrophizing — and rumination (subscale of catastrophizing) on pain intensity, the presence of nature in the urban residential environment reduces pain intensity among community-dwelling middle-aged and older adults living with chronic pain. This finding is consistent with prior evidence linking nature exposure with acute pain reduction (Ulrich, 1984; Diette et al., 2003) and extends our understanding to chronic pain.

These findings-particularly confirmation of the differential hypothesis, that nearby nature moderates the relation between rumination, the most attention-based component of pain catastrophizing and pain intensity, but does not moderate the relations between the other two subscales of pain catastrophizing: helplessness and magnification, and pain intensity-lends credence to the notion that it is through its impact on attention that nature buffers the association between catastrophizing and pain intensity.

This study makes several important contributions relevant to both theory and practice. First, this study extends the findings of prior research that focused on the role of nature in the context of acute pain-to chronic pain. Second, this investigation moves beyond clinical and healthcare settings to consider how nature, a neighborhood resource, might ameliorate the experience of pain intensity. As researchers and practitioners strive to identify strategies to reduce pain intensity experienced by community-dwelling chronic pain sufferers (Reid et al., 2008; Tobias et al., 2014), the nearby natural environment may provide one such opportunity. Importantly, the reported buffering effects of nearby nature are clinically significant-with a reduction of pain intensity of nearly two points (on a 10-point scale) among those with the highest levels of pain catastrophizing (RQ1, Figure 4, Table 2) or pain rumination (RQ2, Figure 5, Table 3). Moreover, the context of this study-a large urban environment-enhances both its ecological validity and practical utility. Globally, an estimated 1.5 billion people experience chronic pain ${ }^{1}$ and according to worldwide statistics, half of the population lives in urban areas, where access to nature may be limited. These findings underscore the importance of ensuringthrough architectural and urban design decisions and policiesthat every resident of our cities has access to nearby nature. Lastly, on a theoretical level, this study begins to integrate findings from diverse arenas. The literature documents that: (1) pain demands attention and impairs performance on cognitive tasks that require attention; (2) nature restores the capacity to direct attention and to recover from cognitive fatigue; (3) and nature stimuli have been employed to reduce the intensity of acute pain; however, these literatures had not previously been integrated, nor applied to issues of chronic pain.

\section{Limitations}

This study is not without limitations. First, because of the heterogeneity of the participant sample (e.g., ethnicity, income, cause, and intensity of chronic pain), generalizability of the results may be limited. Second, many other variables such as anxiety, depression, and social networks that could affect pain catastrophizing were not included in this analysis. Third, due 


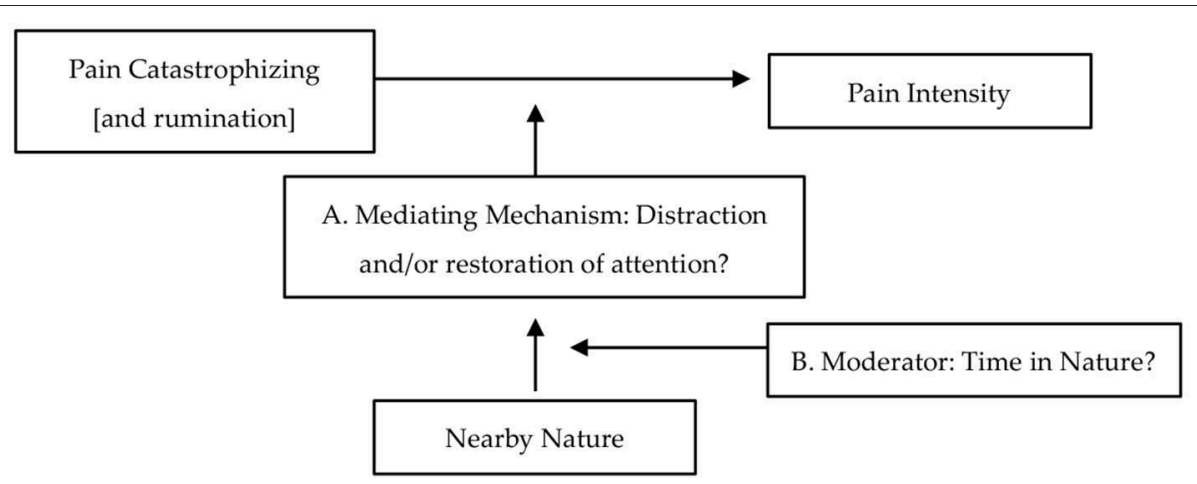

FIGURE 6 | Future research might examine: (A) what mediating mechanism underlies nearby nature's buffering of the pain catastrophizing-pain intensity and rumination-pain intensity associations; and (B) the interaction of nearby nature and time in nature.

to the non-experimental nature of this study, causal conclusions cannot be made. Fourth, although income at the individual level was controlled for in the analyses, neighborhood-level socioeconomic status could be a confounding variable. Fifth, although use of Google Earth images overcomes some limitations of land cover data in urban areas, estimates of nature based on aerial, two-dimensional Google Earth satellite images represent nature visible from an above-ground viewpoint and may not reflect the experience of nature at ground level (Leslie et al., 2010; Sugiyama et al., 2010). Therefore, some variability between imagery and participant experience related to quality, use, and access may have occurred. Sixth, our analyses of time in nature relied on a self-report measure that is subject to reporting biases. Although time in nature served as a control variable in this study, its non-significant effect on pain intensity and absence of a moderating effect on the catastrophizing-pain intensity and rumination-pain intensity associations is somewhat unexpected and may be due to limitations of the self-report measure of time in nature. In addition, small sample size is a limitation. A larger sample, with greater statistical power might yield larger effect sizes.

\section{Future Research}

Further research is needed to better understand the moderating influence of nature on the associations between pain catastrophizing and pain intensity and between pain-related rumination and pain intensity. Although the findings reported here are consistent with prior evidence, the mechanism(s) through which nature affects the pain catastrophizing (and rumination)-pain intensity relation(s) is not clear. While a variety of studies indicate that attention is a key factor in the experience of pain, the notion of distraction may be a relatively superficial perspective. Perhaps the potency of nature to reduce pain is not merely a matter of "distracting" attention, but instead, nature affects attention in a more profound way-by enhancing directed attention capacity-i.e., the ability to focus and concentrate, to control attention and to resist unproductive preoccupations. According to ART, this may be the case. To examine through what mechanism (mediator) nearby nature buffers (moderates) the catastrophizing (and rumination) - pain intensity relation(s) (Figure 6A), future research might employ measures of attentional capacity [such as Digit Span Backwards (Kuo and Sullivan, 2001; Berman et al., 2008) or Attention Network Test (Fan et al., 2002, 2005; Berman et al., 2008)].

In addition, future research might focus on the effects of engaging with nature in addition to the presence of nearby nature. With a more valid and reliable measure of time spent in nature (e.g., objective, rather than self-report), future research might consider how various degrees of engagement with the natural environment affect the catastrophizing (and rumination)-pain intensity relation(s) as well the direct effects of varying degrees of nature engagement on pain intensity. Moreover, future studies might examine a 3-way interaction effect-i.e., pain catastrophizing $\times$ nearby nature $x$ time in nature (Figure 6) with the hypothesis that nearby nature's buffering effect will be bolstered when individuals with chronic pain spend time engaged in nature. Regarding time in nature, future investigations might also consider the context and meaning that pain may have during and after engagement in nature. For example, pain intensity may increase following physical activity in a park and the interpretation of that pain may vary considerably across individuals.

Future studies might also attempt to tease apart distraction of attention from attention restoration in the context of chronic pain by comparing the effect of natural vs. nonnatural stimuli. Such a study might examine non-natural stimuli (hypothesized to distract but not restore attention) to natural stimuli (hypothesized to restore attention). Lastly, intervention research would be a particularly valuable next step. A randomized controlled trial assigning individuals with chronic pain to various levels of nature or to nature vs. nonnature exposure would allow for clearer causal conclusions regarding nature's buffering effect on the pain catastrophizingpain intensity relation.

Other factors that may impact the relationships examined in the current study include an individual's broader socioeconomic niche, the dynamics of specific pain-related behaviors and the interpretation and meaning individuals attribute to their experience of pain, all of which should be the focus of future investigations. 
Our results indicate that the presence of nearby nature buffers the relation between pain-related catastrophizing and pain intensity among commuting-dwelling urban residents who suffer from chronic pain. Moreover, nearby nature moderated the relation between pain-related rumination and pain intensity, but not the relation between helplessness and pain or between magnification and pain, suggesting a central role of attention. These finding may inform the development of pain mitigation strategies to assist people who experience chronic pain.

\section{DATA AVAILABILITY STATEMENT}

The datasets generated for this study are available on request to the corresponding author.

\section{ETHICS STATEMENT}

The studies involving human participants were reviewed and approved by Cornell University. The patients/participants provided their written informed consent to participate in this study.

\section{AUTHOR CONTRIBUTIONS}

AO conceived of the parent study, designed the study, and oversaw data collection. NW conceived of this study. NW and $\mathrm{KR}$ wrote the paper. $\mathrm{KR}$ and $\mathrm{AO}$ conducted the data

\section{REFERENCES}

Arntz, A., Dreessen, L., and De Jong, P. (1994). The influence of anxiety on pain: attentional and attributional mediators. Pain 56, 307-314. doi: 10.1016/0304-3959(94)90169-4

Arntz, A., Dreessen, L., and Merckelbach, H. (1991). Attention, not anxiety, influences pain. Behav. Res. Ther. 29, 41-50. doi: 10.1016/S0005-7967(09)80006-5

Bauer, D. J., and Curran, P. J. (2005). Probing interactions in fixed and multilevel regression: inferential and graphical techniques. Multivariate Behav. Res. 40, 373-400. doi: 10.1207/s15327906mbr4003_5

Berman, M. G., Jonides, J., and Kaplan, S. (2008). The cognitive benefits of interacting with nature. Psychol. Sci. 19, 1207-1212. doi: 10.1111/j.1467-9280.2008.02225.x

Berto, R. (2005). Exposure to restorative environments helps restore attentional capacity. J. Environ. Psychol. 25, 249-259. doi: 10.1016/j.jenvp.2005.07.001

Block, J., and Kremen, A. M. (1996). IQ and ego-resiliency: Conceptual and empirical connections and separateness. J. Pers. Soc. Psychol. 70:349. doi: 10.1037/0022-3514.70.2.349

Bratman, G. N., Hamilton, J. P., Hahn, K. S., Daily, G. C., and Gross, J. J. (2015). Nature experience reduces rumination and subgenual prefrontal cortex activation. Proc. Nat. Acad. Sci. U.S.A. 112, 8567-8572. doi: 10.1073/pnas.1510459112

Capaldi, C. A., Passmore, H.-A., Nisbet, E. K., Zelenski, J. M., and Dopko, R. L. (2015). Flourishing in nature: a review of the benefits of connecting with nature and its application as a wellbeing intervention. Int. J. Wellbeing 5, 1-16. doi: 10.5502/ijw.v5i4.449

Cimprich, B. (1992). Attentional fatigue following breast cancer surgery. Res. Nurs. Health 15, 192-207. doi: 10.1002/nur.4770150306

Cimprich, B., and Ronis, D. L. (2003). An environmental intervention to restore attention in women with newly diagnosed breast cancer. Cancer Nurs. 26, 284-292. doi: 10.1097/00002820-200308000-00005 analysis. MR assisted in study design and oversaw data collection. All authors read, edited, and contributed to the writing of final manuscript.

\section{FUNDING}

The authors gratefully acknowledge financial support from the Translational Research Institute on Pain in Later Life (TRIPLL) of Weil Cornell Medical School and Cornell University, awarded to AO, funding from the John A. Hartford Foundation (an Interdisciplinary Geriatric Health Care Research Center Award) to AO and grants (K24AG053462, P30AG022845) from the National Institute on Aging to MR.

\section{ACKNOWLEDGMENTS}

We also thank Leslie Amanfo of Weill Cornell Medical School for valuable assistance with data collection and Elizabeth Wurth, Julianne Lapsa, and Catherine Schirm of Cornell University for assistance with the development of the nature measurement.

\section{SUPPLEMENTARY MATERIAL}

The Supplementary Material for this article can be found online at: https://www.frontiersin.org/articles/10.3389/fbuil. 2019.00142/full\#supplementary-material

Diette, G. B., Lechtzin, N., Haponik, E., Devrotes, A., and Rubin, H. R. (2003). Distraction therapy with nature sights and sounds reduces pain during flexible bronchoscopy: a complementary approach to routine analygesia. Chest 123, 941-948. doi: 10.1378/chest.123.3.941

Donnellan, M. B., Oswald, F. L., Baird, B. M., and Lucas, R. E. (2006). The miniIPIP scales: tiny-yet-effective measures of the big five factors of personality. Psychol. Assess. 18:192. doi: 10.1037/1040-3590.18.2.192

Eccleston, C. (1995). Chronic pain and distraction: an experimental investigation into the role of sustained and shifting attention in the processing of chronic persistent pain. Behav. Res. Ther. 33, 391-405. doi: 10.1016/0005-7967(94)00057-Q

Eccleston, C., and Crombez, G. (1999). Pain demands attention: a cognitiveaffective model of the interruptive function of pain. Psychol. Bull. 125, 356-366. doi: 10.1037/0033-2909.125.3.356

Faber Taylor, A., and Kuo, F. E. (2009). Children with attention deficits concentrate better after walk in the park. J. Atten. Disord. 12, 402-409. doi: $10.1177 / 1087054708323000$

Faber Taylor, A., Kuo, F. E., and Sullivan, W. C. (2001). Coping with ADD: the surprising connection to green play settings. Environ. Behav. 33, 54-77. doi: $10.1177 / 00139160121972864$

Faber Taylor, A., Kuo, F. E., and Sullivan, W. C. (2002). View of nature and selfdiscipline: evidence from inner city children. J. Environ. Psychol. 22, 49-63. doi: 10.1006/jevp.2001.0241

Fan, J., McCandliss, B. D., Fossella, J., Flombaum, J. I., and Posner, M. I. (2005). The activation of attention networks. Neuroimage 26, 471-479. doi: 10.1016/j.neuroimage.2005.02.004

Fan, J., McCandliss, B. D., Sommer, T., Raz, A., and Posner, M. I. (2002). Testing the efficiency and independence of attentional networks. J. Cogn. Neurosci. 14, 340-347. doi: 10.1162/089892902317361886

Frumkin, H. (2001). Beyond toxicity: human health and the natural environment. Am. J. Prev. Med. 20, 234-240. doi: 10.1016/S0749-3797(00) 00317-2 
Frumkin, H., Bratman, G. N., Breslow, S. J., Cochran, B., Kahn Jr., P. H., Lawler, J. J., et al. (2017). Nature contact and human health: a research agenda. Environ. Health Perspect. 125:075001. doi: 10.1289/EH P1663

Gatchel, R. J. (2004). Comorbidity of chronic pain and mental health disorders: the biopsychosocial perspective. Am. Psychol. 59, 795-805. doi: 10.1037/0003-066X.59.8.795

Groenewegen, P., van den Berg, A., de Vries, S., and Verheij, R. (2006). Vitamin G: the effects of green space on health, well-being, and social safety. BMC Public Health 6:149. doi: 10.1186/1471-2458-6-149

Hartig, T., Mang, M., and Evans, G. W. (1991). Restorative effects of natural environment experiences. Environ. Behav. 23, 3-26. doi: 10.1177/0013916591231001

Hartig, T., Mitchell, R., De Vries, S., and Frumkin, H. (2014). Nature and health. Annu. Rev. Public Health 35, 207-228. doi: 10.1146/annurev-publhealth-032013-182443

Holtzman, S., and DeLongis, A. (2007). One day at a time: the impact of daily satisfaction with spouse responses on pain, negative affect and catastrophizing among individuals with rheumatoid arthritis. Pain 131, 202213. doi: 10.1016/j.pain.2007.04.005

Irvine, K. N., and Warber, S. L. (2002). Greening healthcare: practicing as if the natural environment really mattered. Altern. Ther. Health Med. 8, 76-83.

James, P., Banay, R. F., Hart, J. E., and Laden, F. (2015). A review of the health benefits of greenness. Curr. Epidemiol. Rep. 2, 131-142. doi: 10.1007/s40471-015-0043-7

James, W. (1892). Psychology: A Briefer Course. New York, NY: Holt. doi: $10.1037 / 11630-000$

Jensen, M. P., Turner, J. A., Romano, J. M., and Fisher, L. D. (1999). Comparative reliability and validity of chronic pain intensity measures. Pain 83, 157-162. doi: 10.1016/S0304-3959(99)00101-3

Johannes, C. B., Le, T. K., Zhou, X., Johnston, J. A., and Dworkin, R. H. (2010). The prevalence of chronic pain in United States adults: results of an Internet-based survey. J. Pain 11, 1230-1239. doi: 10.1016/j.jpain.2010.07.002

Kaplan, S. (1995). The restorative benefits of nature: toward an integrative framework. J. Environ. Psychol. 15, 169-182. doi: 10.1016/0272-4944(95)90001-2

Kaplan, S. (2001). Meditation, restoration, and the management of mental fatigue. Environ. Behav. 33, 480-506. doi: 10.1177/00139160121973106

Kjøgx, H., Kasch, H., Zachariae, R., Svensson, P., Jensen, T. S., and Vase, L. (2016). Experimental manipulations of pain catastrophizing influence pain levels in patients with chronic pain and healthy volunteers. Pain 157, 1287-1296. doi: 10.1097/j.pain.0000000000000519

Kline, G. A. (2009). Does a view of nature promote relief from acute pain? J. Holist. Nurs. 27, 159-166. doi: 10.1177/0898010109336138

Kuo, F. E. (2001). Coping with poverty: impacts of environment and attention in the inner city. Environ. Behav. 33, 5-34. doi: 10.1177/00139160121972846

Kuo, F. E., and Sullivan, W. C. (2001). Aggression and violence in the inner city: effects of environment via mental fatigue. Environ. Behav. 33, 543-571. doi: 10.1177/00139160121973124

Lee, D. W. H., Chan, A. C. W., Wong, S. K. H., Fung, T. M. K., Li, A. C. N., Chan, S. K. C., et al. (2004). Can visual distraction decrease the does of patient-controlled sedation required during colonoscopy? A prospective randomized controlled trial. Endoscopy 36, 197-201. doi: 10.1055/s-2004814247

Lembo, T., Fitzgerald, L., Matin, K., Woo, K., Mayer, E. A., and Naliboff, B. D. (1998). Audio and visual stimulation reduces patient discomfort during screening flexible sigmoidoscopy. Am. J. Gastroenterol. 93, 1113-1116. doi: 10.1111/j.1572-0241.1998.00339.x

Leslie, E., Sugiyama, T., Ierodiaconou, D., and Kremera, P. (2010). Perceived and objectively measured greenness of neighbourhoods: are they measuring the same thing? Landsc. Urban Plan. 95, 28-33. doi: 10.1016/j.landurbplan.2009.11.002

Maas, J., de Vries, S., Verheij, R. A., Spreeuwenberg, P., Schellevis, P., and Groenewegen, P. (2009). Morbidity is related to a green living environment. J. Epidemiol. Commun. Health 63, 967-973. doi: 10.1136/jech.2008.079038

Maas, J., Verheij, R. A., Groenewegen, P. P., De Vries, S., and Spreeuwenberg, P. (2006). Green space, urbanity, and health: how strong is the relation? J. Epidemiol. Commun. Health 60, 587-592. doi: 10.1136/jech.2005.043125
McCracken, L. M. (1997). "Attention" to pain in persons with chronic pain: a behavioral approach. Behav. Ther. 28, 271-284. doi: 10.1016/S0005-7894(97)80047-0

Melzack, R. (1999). Pain-an overview. Acta Anaesthesiol. Scand. 43, 880-884. doi: 10.1034/j.1399-6576.1999.430903.x

Miller, A. C., Hickman, L. C., and Lemasters, G. K. (1992). A distraction technique for control of burn pain. J. Burn Care Rehabil. 13, 576-580. doi: 10.1097/00004630-199209000-00012

Mitchell, R. J., and Popham, F. (2007). Greenspace, urbanity and health: relationships in England. J. Epidemiol. Commun. Health 61, 681-683. doi: 10.1136/jech.2006.053553

Moore, E. O. (1981). A prison environment's effect on health care service demands. J. Environ. Syst. 11, 17-34. doi: 10.2190/KM50-WH2K-K2D1-DM69

Nezlek, J. B. (2001). Multilevel random coefficient analyses of event-and intervalcontingent data in social and personality psychology research. Pers. Soc. Psychol. Bull. 27, 771-785. doi: 10.1177/0146167201277001

Ong, A. D., Zautra, A. J., and Reid, M. C. (2010). Psychological resilience predicts decreases in pain catastrophizing through positive emotions. Psychol. Aging 25, 516-523. doi: 10.1037/a0019384

Park, S.-H., Mattson, R., and Kim, E. (2004). "Pain tolerance effects of ornamental plants in a simulated hospital patient room," in Expanding Roles for Horticulture in Improving Human Well-Being and Life Quality, ed D. Relf (Toronto, ON: International Society for Horticultural Science), 241-247. doi: 10.17660/ActaHortic.2004.639.31

Raudenbush, S., Bryk, A., Cheong, Y., Congdon, R., and du Toit, M. (2011). HLM Statistical Software: Version 7. Computer Software.

Reid, M. C., Papaleontiou, M., Ong, A., Breckman, R., Wethington, E., and Pillemer, K. (2008). Self-management strategies to reduce pain and improve function among older adults in community settings: a review of the evidence. Pain Med. 9, 409-424. doi: 10.1111/j.1526-4637.2008.00428.x

Reyes-Gibby, C. C., Aday, L. A., Todd, K. H., Cleeland, C. S., and Anderson, K. O. (2007). Pain in aging community-dwelling adults in the United States: non-Hispanic whites, non-Hispanic blacks, and Hispanics. J. Pain 8, 75-84. doi: 10.1016/j.jpain.2006.06.002

Sugiyama, T., Francis, J., Middleton, N. J., Owen, N., and Giles-Corti, B. (2010). Associations between recreational walking and attractiveness, size, and proximity of neighborhood open spaces. Am. J. Public Health 100, 1752-1757. doi: 10.2105/AJPH.2009.182006

Sullivan, M. J., Bishop, S. R., and Pivik, J. (1995). The pain catastrophizing scale: development and validation. Psychol. Assess. 7, 524-532. doi: 10.1037/1040-3590.7.4.524

Sullivan, M. J., and Neish, N. R. (1998). Catastrophizing, anxiety and pain during dental hygiene treatment. Commun. Dent. Oral Epidemiol. 26, 344-349. doi: 10.1111/j.1600-0528.1998.tb01971.x

Sullivan, M. J., Thorn, B., Haythornthwaite, J. A., Keefe, F., Martin, M., Bradley, L. A., et al. (2001). Theoretical perspectives on the relation between catastrophizing and pain. Clin. J. Pain 17, 52-64. doi: 10.1097/00002508-2001030 00-00008

Takano, T., Nakamura, K., and Watanabe, M. (2002). Urban residential environments and senior citizens longevity in megacity areas: the importance of walkable green spaces. J. Epidemiol. Commun. Health 56, 913-918. doi: $10.1136 /$ jech.56.12.913

Tennessen, C. M., and Cimprich, B. (1995). Views to nature: effects on attention. J. Environ. Psychol. 15, 77-85. doi: 10.1016/0272-4944(95) 90016-0

Tobias, K. R., Lama, S. D., Parker, S. J., Henderson, C. R., Nickerson, A. J., and Reid, M. C. (2014). Meeting the public health challenge of pain in later life: what role can senior centers play? Pain Manage. Nurs. 15, 760-767. doi: 10.1016/j.pmn.2013.07.013

Turk, D. C., and Rudy, T. E. (1992). Cognitive factors and persistent pain: a glimpse into Pandora's box. Cognit. Ther. Res. 16, 99-122. doi: 10.1007/BF011 73484

Ulrich, R. S. (1984). View from window may influence recovery from surgery. Science 224, 420-421. doi: 10.1126/science.6143402

Ulrich, R. S. (1999). "Effects of gardens on health outcomes: theory and research," in Healing Gardens: Therapeutic Benefits and Design Recommendations, eds C. C. Marcus and M. Barnes (New York, NY: John Wiley \& Sons, Inc.), 27-86. 
Verderber, S., and Reuman, D. (1987). Windows, views, and health status in hospital therapeutic environments. J. Architect. Plann. Res. 4, 120-133.

Wells, N. M. (2000). At home with nature: effects of "greenness" on children's cognitive functioning. Environ. Behav. 32, 775-795. doi: 10.1177/00139160021972793

Wells, N. M., and Phalen, K. B. (2018). "Everyday and nearby natural environments," in Environmental Psychology and Human Well-Being: Effects of Built and Natural Settings, ed A. S. Devlin (New York, NY: Academic Press), 221-251. doi: 10.1016/B978-0-12-811481-0.00009-3

Wells, N. M., and Rollings, K. A. (2012). "The natural environment in residential settings: Influences on human health and function," in The Oxford Handbook of Environmental and Conservation Psychology, ed S. D. Clayton (New York, NY: Oxford University Press), 509-523. doi: 10.1093/oxfordhb/9780199733026.013.0027
West, M. J. (1986). Landscape Views and Stress Responses in the Prison Environment. Seattle, WA: University of Washington.

Conflict of Interest: The authors declare that the research was conducted in the absence of any commercial or financial relationships that could be construed as a potential conflict of interest.

Copyright (c) 2019 Wells, Rollings, Ong and Reid. This is an open-access article distributed under the terms of the Creative Commons Attribution License (CC BY).

The use, distribution or reproduction in other forums is permitted, provided the original author(s) and the copyright owner(s) are credited and that the original publication in this journal is cited, in accordance with accepted academic practice. No use, distribution or reproduction is permitted which does not comply with these terms. 